\title{
Bivalirudin or Heparin in Patients Undergoing Invasive Management of Acute Coronary Syndromes
}

\author{
Giuseppe Gargiulo, MD, ${ }^{\mathrm{a}, \mathrm{b}}$ Greta Carrara, Stat, ${ }^{\mathrm{c}}$ Enrico Frigoli, MD, ${ }^{\mathrm{a}}$ Pascal Vranckx, MD, PHD,
} Sergio Leonardi, MD, MHS, ${ }^{\mathrm{e}}$ Nestor Ciociano, PharmD, ${ }^{\mathrm{f}}$ Gianluca Campo, MD, PhD, ${ }^{\mathrm{g}, \mathrm{h}}$ Ferdinando Varbella, MD, ${ }^{\mathrm{i}}$ Paolo Calabrò, MD, $\mathrm{PHD},{ }^{\mathrm{j}}$ Stefano Garducci, MD, ${ }^{\mathrm{k}}$ Alessandro Iannone, $\mathrm{MD},{ }^{\mathrm{l}}$ Carlo Briguori, $\mathrm{MD}, \mathrm{PHD},{ }^{\mathrm{m}}$ Giuseppe Andò, MD, PHD, ${ }^{\mathrm{n}}$ Gabriele Crimi, MD, ${ }^{\mathrm{d}, \mathrm{o}}$ Ugo Limbruno, MD, ${ }^{\mathrm{p}}$ Roberto Garbo, MD, ${ }^{\mathrm{q}}$ Paolo Sganzerla, $\mathrm{MD}$, ${ }^{\mathrm{r}}$ Filippo Russo, MD, ${ }^{\mathrm{s}}$ Alessandro Lupi, MD, ${ }^{\mathrm{t}}$ Bernardo Cortese, MD, ${ }^{\mathrm{u}, \mathrm{v}}$ Arturo Ausiello, MD, ${ }^{\mathrm{w}}$ Salvatore Ierna, MD, ${ }^{\mathrm{X}}$ Giovanni Esposito, MD, PHD, ${ }^{\mathrm{b}}$ Dennis Zavalloni, MD, ${ }^{\mathrm{y}}$ Andrea Santarelli, MD, ${ }^{\mathrm{z}}$ Gennaro Sardella, MD, ${ }^{\text {aa }}$ Simone Tresoldi, MD, ${ }^{\text {bb }}$ Nicoletta de Cesare, MD, ${ }^{\mathrm{cc}}$ Alessandro Sciahbasi, MD, PHD, ${ }^{\text {dd }}$ Antonio Zingarelli, MD, ${ }^{\text {ee }}$ Paolo Tosi, MD, ${ }^{\mathrm{ff}}$ Arnoud van 't Hof, MD, PHD, ${ }^{\text {gg }}$ Elmir Omerovic, MD, ${ }^{\text {hh }}$ Salvatore Brugaletta, MD, ${ }^{\text {ii }}$ Stephan Windecker, MD, ${ }^{\mathrm{a}}$ Marco Valgimigli, $\mathrm{MD}, \mathrm{PHD}^{\mathrm{a}}$

\section{ABSTRACT}

BACKGROUND Contrasting evidence exists on the comparative efficacy and safety of bivalirudin and unfractionated heparin (UFH) in relation to the planned use of glycoprotein IIb/IIla inhibitors (GPIs).

OBJECTIVES This study assessed the efficacy and safety of bivalirudin compared with UFH with or without GPIs in patients with acute coronary syndrome (ACS) who underwent invasive management.

METHODS In the MATRIX (Minimizing Adverse Haemorrhagic Events by Transradial Access Site and Systemic Implementation of AngioX) program, 7,213 patients were randomly assigned to receive either bivalirudin or UFH with or without GPIs at discretion of the operator. The 30-day coprimary outcomes were major adverse cardiovascular events (MACEs) (a composite of death, myocardial infarction, or stroke), and net adverse clinical events (NACEs) (a composite of MACEs or major bleeding).

RESULTS Among 3,603 patients assigned to receive UFH, 781 (21.7\%) underwent planned treatment with GPI before coronary intervention. Bailout use of GPIs was similar between the bivalirudin and UFH groups (4.5\% and $5.4 \%$ ) $(p=0.11)$. At 30 days, the 2 coprimary endpoints of MACEs and NACEs, as well as individual endpoints of mortality, myocardial infarction, stent thrombosis or stroke did not differ among the 3 groups after adjustment. Compared with the UFH and UFH+GPI groups, bivalirudin reduced bleeding, mainly the most severe bleeds, including fatal and nonaccess site-related events, as well as transfusion rates and the need for surgical access site repair. These findings were not influenced by the administered intraprocedural dose of UFH and were confirmed at multiple sensitivity analyses, including the randomly allocated access site.

CONCLUSIONS In patients with ACS, the rates of MACEs and NACEs were not significantly lower with bivalirudin than with UFH, irrespective of planned GPI use. However, bivalirudin significantly reduced bleeding complications, mainly those not related to access site, irrespective of planned use of GPIs. (Minimizing Adverse Haemorrhagic Events by Transradial Access Site and Systemic Implementation of AngioX [MATRIX]; NCT01433627) (J Am Coll Cardiol 2018;71:1231-42) @ 2018 by the American College of Cardiology Foundation.

Listen to this manuscript's audio summary by JACC Editor-in-Chief Dr. Valentin Fuster.
From the a Department of Cardiology, Bern University Hospital, Bern, Switzerland; ${ }^{\mathrm{b}}$ Department of Advanced Biomedical Sciences,

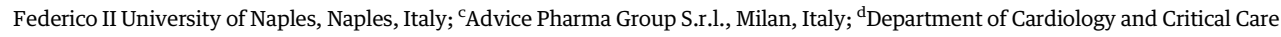
Medicine, Hartcentrum Hasselt, Jessa Ziekenhuis, and Faculty of Medicine and Life Sciences Hasselt University, Hasselt, Belgium; 'SC Terapia Intensiva Cardiologica, Fondazione IRCCS Policlinico San Matteo, Pavia, Italy; ${ }^{\mathrm{f} E U S T R A T E G Y ~ A s s o c i a t i o n, ~ F o r l i ', ~ I t a l y ; ~}$ ${ }^{\mathrm{g} C a r d i o v a s c u l a r}$ Institute, Azienda Ospedaliero-Universitaria di Ferrara, Cona (FE), Italy; ${ }^{\mathrm{h}}$ Maria Cecilia Hospital, GVM Care and Research, Cotignola (RA), Italy; ${ }^{\mathrm{i} C a r d i o l o g y}$ Unit, Ospedali Riuniti di Rivoli, ASL Torino 3, Turin, Italy; ${ }^{\mathrm{j} D i v i s i o n}$ of Cardiology, Department of Cardiothoracic Sciences, University of Campania "Luigi Vanvitelli", Naples, Italy; ${ }^{k}$ Struttura complessa di Cardiologia ASST di Vimercate, Italy; ${ }^{\mathrm{l}}$ Department of Cardiology, ASL3 Ospedale Villa Scassi, Genoa, Italy; ${ }^{\mathrm{m}}$ Interventional 
ABBREVIATIONS AND ACRONYMS

\section{ACS = acute coronary syndrome(s)}

CABG = coronary artery bypass grafting

GPI = glycoprotein IIb/IIla inhibitor

MACE = major adverse cardiovascular event

NACE = net adverse clinical event

NSTE-ACS $=$ non ST-segment elevation acute coronary syndrome(s)

$\mathrm{PCI}=$ percutaneous coronary intervention

ST $=$ stent thrombosis

TIMI = Thrombolysis In Myocardial Infarction

$\mathrm{UFH}=$ unfractionated heparin
$\mathrm{T}$ he most effective antithrombotic therapy in patients with an acute coronary syndrome (ACS) who are undergoing a percutaneous coronary intervention (PCI) remains strongly debated (1-3). Unfractionated heparin (UFH) (with or without planned glycoprotein IIb/IIIa inhibitors [GPIs]) and bivalirudin are 2 of the most commonly used antithrombotic strategies and have been compared in different trials since the 1990s (4). Conflicting data have accumulated since then, so that the comparative safety and effectiveness profile of bivalirudin compared with UFH alone in current practice remains unclear.

Although some trials, including EUROMAX (European Ambulance Acute Coronary Syndrome Angiography Trial) $(5,6)$ and BRIGHT (Bivalirudin in Acute Myocardial Infarction vs Heparin and GPI Plus Heparin Trial) (7), have shown benefits in terms of major bleeding reduction related to bivalirudin use, irrespective of GPI use in the UFH arm, the HEAT-PPCI (How Effective are Antithrombotic Therapies in Primary Percutaneous Coronary Intervention) and the most recent VALIDATESWEDEHEART (Bivalirudin versus Heparin in
ST-Segment and Non-ST-Segment Elevation Myocardial Infarction in Patients on Modern Antiplatelet Therapy in the Swedish Web System for Enhancement and Development of Evidence-based Care in Heart Disease Evaluated according to Recommended Therapies Registry Trial) studies showed that heparin alone did not increase bleeding events compared with bivalirudin $(8,9)$. Because planned use of GPIs in patients who receive UFH has been reduced, this discrepancy is notable.

$$
\text { SEE PAGE } 1243
$$

Therefore, we pre-specified to examine the comparative efficacy and safety profile of bivalirudin compared with UFH alone or with UFH+GPI in the context of the largest contemporary trial to assess the value of bivalirudin in an all-comer ACS population and the only study that allocated access site by random selection.

\section{METHODS}

STUDY DESIGN. The MATRIX (Minimizing Adverse Haemorrhagic Events by Transradial Access Site and Systemic Implementation of AngioX) antithrombin study is a randomized, multicenter trial that compared bivalirudin (the use of GPIs was restricted

Cardiology Unit, Clinica Mediterranea, Naples, Italy; "Azienda Ospedaliera Universitaria Policlinico “Gaetano Martino”, University

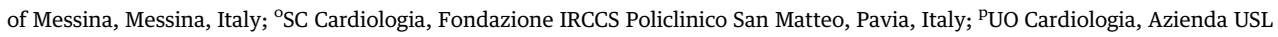
Toscana Sudest, Grosseto, Italy; ${ }^{\mathrm{q}}$ Interventional Cardiology Unit, Ospedale San Giovanni Bosco, Turin, Italy; ${ }^{\mathrm{r}}$ ASST Bergamo ovest, Ospedale di Treviglio (BG), Italy; ${ }^{\text {s} C a r d i o v a s c u l a r ~ I n t e r v e n t i o n a l ~ U n i t, ~ C a r d i o l o g y ~ D e p a r t m e n t, ~ S . A n n a ~ H o s p i t a l, ~ C o m o, ~ I t a l y ; ~}$

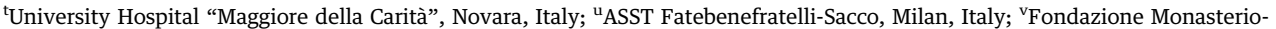
CNR-Regione Toscana, Toscana, Italy; ${ }^{\text {w}}$ Casa di Cura Villa Verde, Taranto, Italy; ${ }^{\mathrm{x}}$ Simple Departmental Emodynamic Structure, Ospedale Sirai-Carbonia, Carbonia, Italy; ${ }^{y}$ Humanitas Research Hospital, IRCCS, Rozzano, Italy; ${ }^{z}$ Cardiovascular Department, Infermi Hospital, Rimini, Italy; ${ }^{\text {aa }}$ Department of Cardiovascular, Respiratory, Nephrologic, Anesthesiologic and Geriatric Sciences, Policlinico Umberto I, "Sapienza”, University of Rome, Rome, Italy; ${ }^{b}$ Struttura complessa di Emodinamica, ASST Monza,

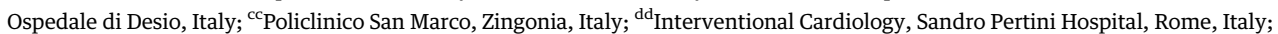
${ }^{e}$ Clinic of Cardiovascular Disease, IRCCS Policlinico San Martino, Genoa, Italy; ${ }^{\mathrm{ff}}$ Mater Salutis Hospital-Legnago, Verona, Italy;

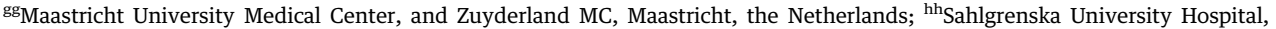

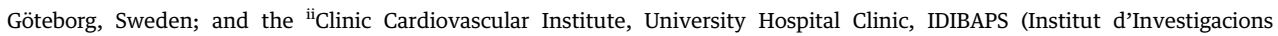
Biomèdiques August Pi i Sunyer), Barcelona, Spain. The trial was sponsored by the Società Italiana di Cardiologia Invasiva (GISE, a non-profit organization), which received grant support from The Medicines Company and TERUMO. This substudy did not receive any direct or indirect funding. Dr. Gargiulo has received research grant support from the Cardiopath PhD program. Dr. Vranckx has received speaking or consulting fees from Bayer Health Care and Daiichi-Sankyo. Dr. Leonardi has received grants and personal fees from AstraZeneca; and personal fees from Chiesi, Daiichi-Sankyo, and The Medicines Company. Dr. Varbella has received speaking or consulting fees from Boeringher Ingelheim, Daiichi-Sankyo, Bayer, Pfizer, AstraZeneca, OrbusNeich, Biosensors, AbbottVascular, Amgen, and Bristol-Myers Squibb; and has received grants from Medtronic, Boston Scientific, Abbott, St. Jude, Biosensors, CID Alvimedica, and Abbott Vascular. Dr. Andò has received nonfinancial support from Terumo during the study; personal fees from Daiichi-Sankyo, Pfizer, and AstraZeneca; and personal fees and nonfinancial support from Bayer. Dr. Cortese has received research grants from AB Medica, Abbott, St. Jude Medical, and Stentys; and personal fees from Abbott, AstraZeneca, Daiichi-Sankyo, and Eli Lilly and Stentys. Dr Sciahbasi has served as advisory board member for Bayer HealthCare. Dr van’t Hof has received speaker fees from The Medicines Company; has received unrestricted grants from Medtronic and AstraZeneca; and has served as Executive Board member of the EUROMAX trial. Dr. Omervoic has been a member of the advisory board for Boston Scientific; and has received a research grant from AstraZeneca. Dr. Windecker has received research grants from Abbott, Amgen, Bracco, Boston Scientific, Biotronick, St. Jude, and Terumo. Dr. Valgimigli has received grants from The Medicines Company, Terumo, and AstraZeneca; and has received personal fees from Terumo, St. Jude Vascular, and Abbott Vascular. All other authors have reported that they have no relationships relevant to the contents of this paper to disclose. 
to bailout conditions) with UFH (use of GPI was left to the discretion of the investigator) in 7,213 patients with ACS with or without ST-segment elevation, in whom PCI was planned. This was 1 of 3 trials of the MATRIX program (NCT01433627), as previously described $(1,10)$.

STUDY PATIENTS. Patients with non-ST-segment elevation acute coronary syndrome (NSTE-ACS) were eligible if they had a history consistent with new or worsening cardiac ischemia that occurred while they were at rest or with minimal activity within 7 days before randomization, and met at least 2 high-risk criteria among the following: 1) age of 60 years or older, elevation of cardiac biomarkers, or electrocardiographic changes compatible with ischemia; and 2) if they were considered to be candidates for PCI after completion of coronary angiography. Patients with STsegment elevation myocardial infarction (STEMI) were eligible if they presented within $12 \mathrm{~h}$ of the onset of symptoms or between 12 and $24 \mathrm{~h}$ after symptom onset if there was evidence of continued ischemia or previous fibrinolytic treatment. The main inclusion and exclusion criteria were previously reported $(1,10)$. All patients provided written informed consent.

STUDY PROTOCOL AND RANDOMIZATION. Using a computer-generated random sequence, we randomized patients in a 1:1 ratio to receive bivalirudin or $\mathrm{UFH}$, with a random block size stratified by the type of ACS (i.e., with ST-segment elevation vs. without ST-segment elevation) intended for or ongoing use of a $\mathrm{P}_{2} \mathrm{Y}_{12}$ inhibitor (clopidogrel vs. ticagrelor or prasugrel), and study site. Patients with STEMI underwent randomization before coronary angiography; patients with NSTE-ACS underwent randomization immediately after completion of angiography but before the start of PCI.

All interventions were administered in an openlabel fashion. Bivalirudin was given according to the product labeling, with a bolus of $0.75 \mathrm{mg} / \mathrm{kg}$ of body weight, followed immediately by an infusion of $1.75 \mathrm{mg} / \mathrm{kg} / \mathrm{h}$ until completion of PCI. Receiving a postPCI bivalirudin infusion or no post-PCI infusion was randomly determined (MATRIX treatment duration). In those assigned to bivalirudin prolongation, the choice between 2 regimens (full dose for up to $4 \mathrm{~h}$ or reduced dose of $0.25 \mathrm{mg} / \mathrm{kg} / \mathrm{h}$ for at least $6 \mathrm{~h}$ ) was made at the discretion of the treating physicians. UFH was administered at a dose of 70 to $100 \mathrm{U}$ or 50 to $70 \mathrm{U} / \mathrm{kg}$ in patients who did not receive or received GPI, respectively. Subsequent UFH dose adjustment based on the activated clotting time was left to the discretion of the treating physicians. A GPI could be administered before PCI in all patients in the UFH group based on judgment of the treating physician, but the drug was to be administered in the bivalirudin group only to patients who had periprocedural ischemic complications (i.e., no-reflow or giant thrombus) after PCI. The use of other medications was allowed according to professional guidelines.

FOLLOW-UP AND STUDY OUTCOMES. Clinical follow-up was performed at 30 days. Two coprimary 30-day composite outcomes were pre-specified: major adverse cardiovascular events (MACEs), defined as the composite of all-cause mortality, myocardial infarction (MI), or stroke; and net adverse clinical events (NACEs), defined as the composite of MACEs or major bleeding not related to coronary artery bypass graft (CABG) (Bleeding Academic Research Consortium type 3 or 5). Secondary outcomes included each component of the composite outcomes, cardiovascular mortality, and stent thrombosis (ST). Bleeding was also assessed and adjudicated on the basis of the TIMI (Thrombolysis In Myocardial Infarction) and GUSTO (Global Utilization of Streptokinase and Tissue Plasminogen Activator) scales. Stent thrombosis was defined as the definite or probable occurrence of a stent-related thrombotic event according to the Academic Research Consortium classification. All outcomes were pre-specified. An independent clinical events committee blinded to treatment allocation adjudicated all suspected events.

STATISTICAL ANALYSIS. The trial was powered for superiority on the 2 coprimary composite outcomes at 30 days, expecting a rate reduction of $30 \%$ that corresponded to a rate ratio of 0.70 .

All analyses were performed per intention-to-treat principle, including all patients in the analysis based on the allocated access. Events up to 30 days post-randomization were considered. We analyzed primary and secondary outcomes as time to first event using the Mantel-Cox method, accompanied by log-rank tests to calculate corresponding 2-sided $\mathrm{p}$ values. We did not perform any adjustments for multiple comparisons but set the alpha error at $2.5 \%$ to correct for the 2 coprimary outcomes. We analyzed secondary outcomes with a 2-sided alpha set at $5 \%$ to allow conventional interpretation of results. Survival curves were constructed using Kaplan-Meier estimates. We performed stratified analyses according to the dosage of heparin used or to access site (radial or femoral) and we estimated possible interaction terms across comparisons.

Whether the attribution to bivalirudin and UFH arms was randomized, the planned use of GPI was only allowed in the UFH arm and was left to the discretion of the physician. Because of the 
nonrandomized nature of the planned GPI use in the UFH arm, clinical outcomes were adjusted for confounders. A multivariable logistic model was used to obtain adjusted analyses, and the variables included were age, sex, body mass index, type of ACS, center, diabetes, smoking, hypertension, previous MI, previous CABG, previous stroke and/or transient ischemic attack (TIA), peripheral vascular disease, Killip class, previous lytic therapy, creatinine, intraaortic balloon pump, heparin use before arrival at the catheterization laboratory, full procedural success, duration of the procedure, treated vessel, SYNTAX (Synergy Between Percutaneous Coronary Intervention with Taxus and Cardiac Surgery) score, proximal location of lesion, large vessel caliber (defined by using a stent $\geq 3 \mathrm{~mm}$ or post-dilatation balloon $\geq 3.5 \mathrm{~mm}$ ), $\geq 1$ complex lesion, thrombus in the treated lesion, TIMI flow 0 to 1 before PCI, and clopidogrel at discharge.

As a sensitivity analysis, a propensity score was calculated to minimize any selection bias due to the differences in clinical characteristics between the 2 treatment groups (i.e., UFH alone and UFH + GPI). For each patient in the UFH arm, a propensity score that indicated the likelihood of receiving GPI was calculated by the use of a nonparsimonious multivariable logistic regression. A propensity score that indicated the predicted probability of receiving a specific treatment conditional on the observed covariates was then calculated from the logistic equation for each patient. Then, the formula was also applied for patients in the bivalirudin arm (in which per-protocol planned GPI was not allowed). The following variables were included: age, sex, body mass index, type of ACS, center, diabetes, smoking, family history of coronary artery disease, hypertension, hypercholesterolemia, previous MI, previous PCI, previous stroke and/or TIA, peripheral vascular disease, chronic obstructive pulmonary disease, Killip class, cardiac arrest, left ventricular ejection fraction, creatinine, treated vessel, SYNTAX score, proximal location of the lesion, large and/or small vessel caliber, $\geq 1$ complex lesion, thrombus in the treated lesion, TIMI flow o to 1 before PCI, medication in the catheterization laboratory (fondaparinux, enoxaparin, beta-blockers, ticagrelor, clopidogrel), and lesions treated and stented per patients. The individual propensity score was incorporated into the adjustment model to compare outcomes. In addition, to reduce the effect of treatment selection bias and potential confounding related to these observational comparisons, we performed rigorous adjustment for significant differences in the baseline characteristics of patients with propensity score matching using the following algorithm: 1:1 optimal match with a 0.1 SD caliper and no replacement. All analyses were performed using the STATA version 14.1 (StataCorp, College Station, Texas) and R (R Foundation, Vienna, Austria) statistical packages.

\section{RESULTS}

PATIENTS. The MATRIX-antithrombin trial enrolled 7,213 patients with ACS from 78 centers in Italy, the Netherlands, Spain, and Sweden between October 2011 and November 2014. Of these patients, 3,610 patients were assigned to receive bivalirudin and 3,603 were assigned to receive $\mathrm{UFH}$, of whom 2,822 received UFH without planned GPI infusion (UFH alone group) and 781 patients underwent planned treatment with GPI before coronary intervention (UFH+GPI group). In the bivalirudin and UFH groups, a similar proportion of patients received bailout use of GPIs during treatment $(4.5 \%$ and $5.4 \%$, respectively; $\mathrm{p}=0.11$ ).

Clinical and procedural characteristics as well as choice of concomitant medications during hospitalization or at discharge were imbalanced among the 3 groups (Online Tables 1 to 3 ).

Compared with UFH alone, patients with planned GPI were younger, more frequently male, smokers, and had STEMI or cardiac arrest at presentation, but less frequently had a history of diabetes, hypertension, MI, PCI, CABG, stroke/TIA, chronic obstructive pulmonary disease, peripheral vascular disease, or renal dysfunction (Online Table 1). Patients who received planned GPI infusion experienced a longer procedural time, despite more frequently receiving single-vessel intervention. They more frequently required intra-aortic balloon pumps; recanalization of occluded, proximally located, and thrombuscontaining lesion(s); and required larger stent diameters and longer overall stent length (Online Table 2).

CLINICAL OUTCOMES. Unadjusted and adjusted comparisons for the 30-day outcomes across the groups are shown in Tables 1 to 3 . After multivariable or propensity-score adjustment, the 2 coprimary endpoints of MACEs and NACEs did not differ among the 3 groups (Figure 1, Central Illustration, Tables 1 to 3 ).

Similarly, there were no within-groups differences with respect to the individual endpoints of mortality, MI, ST, or stroke (Tables 1 to 3). However, bivalirudin remained associated with reduced risks of bleeding due to lower rates of the most severe occurrences, including fatal and nonaccess site-related, mainly gastrointestinal events compared with UFH alone and to lower risks of both gastrointestinal and 
TABLE 1 Clinical Outcomes up to 30 Days in Bivalirudin Versus UFH Alone

\begin{tabular}{|c|c|c|c|c|c|c|c|c|c|}
\hline & $\begin{array}{c}\text { All } \\
(\mathrm{N}=\mathbf{7 , 2 1 3})\end{array}$ & $\begin{array}{l}\text { Bivalirudin } \\
(\mathbf{n}=\mathbf{3 , 6 1 0})\end{array}$ & $\begin{array}{l}\text { UFH Alone } \\
(\mathbf{n}=\mathbf{2 , 8 2 2})\end{array}$ & $\begin{array}{c}\text { Unadjusted } \\
\text { Rate Ratio } \\
(95 \% \mathrm{Cl})\end{array}$ & p Value & $\begin{array}{c}\text { Multivariable } \\
\text { Adjusted Rate Ratio } \\
(95 \% \mathrm{Cl})\end{array}$ & p Value & $\begin{array}{c}\text { Propensity } \\
\text { Score Adjusted } \\
\text { Rate Ratio } \\
(95 \% \mathrm{Cl})\end{array}$ & p Value \\
\hline Death, MI, stroke & $762(10.6)$ & $371(10.3)$ & $327(11.6)$ & $0.89(0.76-1.03)$ & 0.113 & $1.02(0.85-1.22)$ & 0.843 & $0.96(0.81-1.13)$ & 0.618 \\
\hline Death, MI, stroke, BARC 3 or 5 & $845(11.7)$ & $401(11.1)$ & $362(12.8)$ & $0.87(0.75-1.00)$ & 0.047 & $0.96(0.81-1.14)$ & 0.664 & $0.92(0.78-1.07)$ & 0.282 \\
\hline $\begin{array}{l}\text { Death, MI, stroke, } \\
\text { BARC } 3 \text { or } 5 \text {, TVR, ST }\end{array}$ & 860 (11.9) & $410(11.4)$ & $367(13.0)$ & $0.87(0.76-1.01)$ & 0.059 & $0.97(0.82-1.15)$ & 0.739 & $0.92(0.79-1.08)$ & 0.309 \\
\hline Death & $142(2.0)$ & $59(1.6)$ & $65(2.3)$ & $0.71(0.5-1.01)$ & 0.055 & $0.91(0.43-1.94)$ & 0.805 & $0.71(0.48-1.04)$ & 0.08 \\
\hline Cardiovascular death & $136(1.9)$ & $56(1.6)$ & $63(2.2)$ & $0.69(0.48-1.00)$ & 0.046 & $1.01(0.45-2.28)$ & 0.976 & $0.69(0.47-1.03)$ & 0.072 \\
\hline MI & $610(8.5)$ & $307(8.5)$ & $258(9.1)$ & $0.93(0.79-1.1)$ & 0.391 & $1.03(0.85-1.24)$ & 0.771 & $1.03(0.86-1.23)$ & 0.778 \\
\hline Stroke & $29(0.4)$ & $13(0.4)$ & $15(0.5)$ & $0.68(0.32-1.42)$ & 0.301 & $0.56(0.24-1.28)$ & 0.17 & $0.58(0.27-1.28)$ & 0.178 \\
\hline TIA & $14(0.2)$ & $5(0.1)$ & $7(0.2)$ & $0.56(0.18-1.76)$ & 0.313 & $0.91(0.21-4.02)$ & 0.905 & $0.73(0.22-2.42)$ & 0.611 \\
\hline TVR & $87(1.2)$ & $52(1.4)$ & $25(0.9)$ & $1.63(1.01-2.62)$ & 0.044 & $1.50(0.90-2.51)$ & 0.118 & $1.40(0.85-2.29)$ & 0.186 \\
\hline ST definite & $57(0.8)$ & $36(1.0)$ & $15(0.5)$ & $1.88(1.03-3.43)$ & 0.037 & $1.77(0.91-3.41)$ & 0.091 & $1.56(0.84-2.91)$ & 0.163 \\
\hline Acute & $33(0.5)$ & $20(0.6)$ & $9(0.3)$ & $1.74(0.79-3.82)$ & 0.164 & $1.85(0.76-4.50)$ & 0.178 & $1.45(0.64-3.27)$ & 0.369 \\
\hline Subacute & $24(0.3)$ & $16(0.4)$ & $6(0.2)$ & $2.08(0.82-5.33)$ & 0.117 & $1.56(0.57-4.25)$ & 0.386 & $1.72(0.65-4.54)$ & 0.275 \\
\hline ST definite/probable & $80(1.1)$ & $45(1.2)$ & $26(0.9)$ & $1.35(0.83-2.19)$ & 0.218 & $1.57(0.88-2.83)$ & 0.129 & $1.32(0.79-2.23)$ & 0.291 \\
\hline Acute & $38(0.5)$ & $22(0.6)$ & $12(0.4)$ & $1.43(0.71-2.90)$ & 0.313 & $1.93(0.80-4.68)$ & 0.144 & $1.25(0.59-2.66)$ & 0.562 \\
\hline Subacute & $42(0.6)$ & $23(0.6)$ & $14(0.5)$ & $1.28(0.66-2.50)$ & 0.459 & $1.18(0.52-2.64)$ & 0.692 & $1.39(0.68-2.85)$ & 0.371 \\
\hline Bleeding & $873(12.1)$ & $391(10.8)$ & $345(12.2)$ & $0.89(0.77-1.02)$ & 0.101 & $0.84(0.71-1.00)$ & 0.044 & $0.85(0.72-1.00)$ & 0.043 \\
\hline BARC 1 & 427 (5.9) & $190(5.3)$ & $170(6.0)$ & $0.87(0.71-1.07)$ & 0.201 & $0.83(0.66-1.04)$ & 0.10 & $0.84(0.68-1.05)$ & 0.126 \\
\hline BARC 2 & $304(4.2)$ & $151(4.2)$ & $107(3.8)$ & $1.10(0.86-1.41)$ & 0.437 & $1.03(0.79-1.34)$ & 0.827 & $1.09(0.84-1.42)$ & 0.505 \\
\hline BARC 3 & $116(1.6)$ & $44(1.2)$ & $50(1.8)$ & $0.69(0.46-1.03)$ & 0.069 & $0.69(0.45-1.07)$ & 0.096 & $0.65(0.42-0.99)$ & 0.045 \\
\hline BARC 3a & $62(0.9)$ & $24(0.7)$ & $24(0.9)$ & $0.78(0.44-1.38)$ & 0.392 & $1.03(0.55-1.90)$ & 0.935 & $0.76(0.42-1.37)$ & 0.361 \\
\hline BARC 3b & $49(0.7)$ & $16(0.4)$ & $25(0.9)$ & $0.50(0.27-0.94)$ & 0.027 & $0.42(0.22-0.81)$ & 0.01 & $0.47(0.24-0.90)$ & 0.024 \\
\hline BARC $3 c$ & $5(0.1)$ & $4(0.1)$ & $1(0)$ & $3.13(0.35-27.98)$ & 0.282 & - & - & $2.17(0.23-20.59)$ & 0.499 \\
\hline BARC 4 & $5(0.1)$ & $1(0)$ & $4(0.1)$ & $0.20(0.02-1.75)$ & 0.104 & - & - & $0.31(0.03-2.98)$ & 0.31 \\
\hline BARC 5 & $21(0.3)$ & $5(0.1)$ & $14(0.5)$ & $0.28(0.10-0.78)$ & 0.009 & - & - & $0.20(0.06-0.62)$ & 0.006 \\
\hline BARC 5a & $15(0.2)$ & $4(0.1)$ & $9(0.3)$ & $0.35(0.11-1.13)$ & 0.065 & $0.26(0.03-2.08)$ & 0.206 & $0.24(0.06-0.96)$ & 0.043 \\
\hline BARC 5b & $6(0.1)$ & $1(0)$ & $5(0.2)$ & $0.16(0.02-1.34)$ & 0.051 & - & - & $0.12(0.01-1.12)$ & 0.063 \\
\hline BARC 3 or 5 & 137 (1.9) & $49(1.4)$ & $64(2.3)$ & $0.60(0.41-0.87)$ & 0.006 & $0.65(0.43-0.99)$ & 0.043 & $0.55(0.37-0.81)$ & 0.003 \\
\hline BARC 3 or 5 access site & $51(0.7)$ & $19(0.5)$ & $23(0.8)$ & $0.65(0.35-1.19)$ & 0.155 & $0.65(0.34-1.24)$ & 0.19 & $0.66(0.35-1.26)$ & 0.209 \\
\hline BARC 3 or 5 nonaccess site & $86(1.2)$ & $30(0.8)$ & $41(1.5)$ & $0.57(0.36-0.92)$ & 0.018 & $0.64(0.37-1.12)$ & 0.117 & $0.49(0.30-0.81)$ & 0.005 \\
\hline BARC 2,3 or 5 & $441(6.1)$ & $200(5.5)$ & $171(6.1)$ & $0.91(0.75-1.12)$ & 0.389 & $0.90(0.72-1.13)$ & 0.361 & $0.88(0.71-1.10)$ & 0.257 \\
\hline BARC 2,3 or 5 access site & $237(3.3)$ & $105(2.9)$ & $98(3.5)$ & $0.84(0.64-1.10)$ & 0.206 & $0.81(0.61-1.09)$ & 0.165 & $0.86(0.65-1.14)$ & 0.298 \\
\hline BARC 2,3 or 5 nonaccess site & $204(2.8)$ & $95(2.6)$ & $73(2.6)$ & $1.02(0.75-1.38)$ & 0.912 & $1.04(0.74-1.46)$ & 0.83 & $0.92(0.66-1.27)$ & 0.599 \\
\hline TIMI major & $49(0.7)$ & $16(0.4)$ & $26(0.9)$ & $0.48(0.26-0.9)$ & 0.019 & $0.39(0.18-0.83)$ & 0.015 & $0.38(0.20-0.74)$ & 0.005 \\
\hline TIMI minor & $50(0.7)$ & $17(0.5)$ & $20(0.7)$ & $0.66(0.35-1.27)$ & 0.212 & $0.79(0.40-1.56)$ & 0.492 & $0.65(0.33-1.29)$ & 0.216 \\
\hline TIMI major/minor & $99(1.4)$ & $33(0.9)$ & $46(1.6)$ & $0.56(0.36-0.88)$ & 0.010 & $0.57(0.34-0.95)$ & 0.03 & $0.49(0.30-0.79)$ & 0.004 \\
\hline GUSTO severe & $42(0.6)$ & $16(0.4)$ & $20(0.7)$ & $0.63(0.32-1.21)$ & 0.158 & $0.67(0.29-1.54)$ & 0.35 & $0.47(0.23-0.95)$ & 0.037 \\
\hline GUSTO moderate & $42(0.6)$ & $16(0.4)$ & $17(0.6)$ & $0.74(0.37-1.46)$ & 0.376 & $0.92(0.43-1.96)$ & 0.823 & $0.79(0.39-1.62)$ & 0.526 \\
\hline GUSTO mild & 784 (10.9) & 358 (9.9) & $304(10.8)$ & $0.92(0.79-1.07)$ & 0.289 & $0.86(0.72-1.02)$ & 0.081 & $0.89(0.75-1.05)$ & 0.173 \\
\hline GUSTO moderate/severe & $84(1.2)$ & $32(0.9)$ & $37(1.3)$ & $0.68(0.42-1.09)$ & 0.103 & $0.79(0.45-1.37)$ & 0.398 & $0.61(0.37-1.01)$ & 0.053 \\
\hline $\begin{array}{l}\text { Composite of surgical access site } \\
\text { repair and blood transfusion }\end{array}$ & $103(1.4)$ & $36(1.0)$ & $51(1.8)$ & $0.55(0.36-0.85)$ & 0.006 & $0.52(0.32-0.84)$ & 0.008 & $0.58(0.37-0.91)$ & 0.018 \\
\hline Surgical access site repair & $17(0.2)$ & $5(0.1)$ & $8(0.3)$ & $0.49(0.16-1.49)$ & 0.199 & $0.52(0.15-1.74)$ & 0.289 & $0.58(0.18-1.84)$ & 0.354 \\
\hline Blood transfusion & $94(1.3)$ & $31(0.9)$ & $47(1.7)$ & $0.52(0.33-0.81)$ & 0.004 & $0.47(0.28-0.79)$ & 0.004 & $0.54(0.33-0.87)$ & 0.011 \\
\hline \multicolumn{10}{|l|}{ Distribution of BARC 3 or 5} \\
\hline Intracranial bleeding & $7(0.1)$ & $4(0.1)$ & $3(0.1)$ & $1.04(0.23-4.66)$ & 0.957 & $0.48(0.06-3.92)$ & 0.496 & $0.83(0.18-3.91)$ & 0.815 \\
\hline Pericardial bleeding & $28(0.4)$ & $11(0.3)$ & $14(0.5)$ & $0.61(0.28-1.35)$ & 0.222 & $0.76(0.24-2.40)$ & 0.637 & $0.49(0.21-1.13)$ & 0.094 \\
\hline Gastrointestinal bleeding & $27(0.4)$ & $6(0.2)$ & $16(0.6)$ & $0.29(0.11-0.75)$ & 0.006 & $0.31(0.12-0.85)$ & 0.023 & $0.26(0.09-0.71)$ & 0.008 \\
\hline Genito-urinary bleeding & $12(0.2)$ & $5(0.1)$ & $2(0.1)$ & $1.95(0.38-10.07)$ & 0.415 & $2.80(0.40-19.41)$ & 0.298 & $2.04(0.39-10.64)$ & 0.399 \\
\hline Access site bleeding & $49(0.7)$ & $19(0.5)$ & $22(0.8)$ & $0.68(0.37-1.25)$ & 0.207 & $0.70(0.36-1.33)$ & 0.27 & $0.69(0.36-1.33)$ & 0.267 \\
\hline Other bleeding & $10(0.1)$ & $3(0.1)$ & $5(0.2)$ & $0.47(0.11-1.96)$ & 0.288 & $1.17(0.12-11.52)$ & 0.891 & $0.37(0.07-1.90)$ & 0.232 \\
\hline
\end{tabular}

Values are $\mathrm{n}(\%)$ unless otherwise indicated.

$\mathrm{BARC}=$ Bleeding Academic Research Consortium; $\mathrm{Cl}=$ confidence interval; GUSTO = Global Utilization of Streptokinase and Tissue Plasminogen Activator; MI = myocardial infarction; ST = stent thrombosis; TIA = transient ischemic attack; TIMI = Thrombolysis In Myocardial Infarction; TVR = target vessel revascularization; UFH $=$ unfractionated heparin. 


\begin{tabular}{|c|c|c|c|c|c|c|c|c|c|}
\hline & $\begin{array}{c}\text { All } \\
(\mathrm{N}=\mathbf{7 , 2 1 3})\end{array}$ & $\begin{array}{l}\text { Bivalirudin } \\
(\mathrm{n}=\mathbf{3}, 610)\end{array}$ & $\begin{array}{l}\text { UFH }+ \text { GPI } \\
(n=781)\end{array}$ & $\begin{array}{l}\text { Unadjusted } \\
\text { Rate Ratio } \\
(95 \% \mathrm{Cl})\end{array}$ & p Value & $\begin{array}{c}\text { Multivariable } \\
\text { Adjusted Rate Ratio } \\
(95 \% \mathrm{CI})\end{array}$ & p Value & $\begin{array}{c}\text { Propensity } \\
\text { Score Adjusted } \\
\text { Rate Ratio } \\
(95 \% \mathrm{Cl})\end{array}$ & p Value \\
\hline Death, MI, stroke & 762 (10.6) & $371(10.3)$ & $64(8.2)$ & $1.25(0.96-1.64)$ & 0.094 & $0.99(0.70-1.39)$ & 0.947 & $0.95(0.70-1.29)$ & 0.731 \\
\hline Death, MI, stroke, BARC 3 or 5 & $845(11.7)$ & $401(11.1)$ & $82(10.5)$ & $1.06(0.83-1.34)$ & 0.642 & $0.80(0.59-1.09)$ & 0.153 & $0.79(0.60-1.05)$ & 0.108 \\
\hline $\begin{array}{l}\text { Death, MI, stroke, } \\
\text { BARC } 3 \text { or } 5, T V R \text {, ST }\end{array}$ & 860 (11.9) & $410(11.4)$ & $83(10.6)$ & $1.07(0.84-1.35)$ & 0.581 & $0.81(0.60-1.10)$ & 0.170 & $0.81(0.62-1.07)$ & 0.146 \\
\hline Death & $142(2.0)$ & $59(1.6)$ & $18(2.3)$ & $0.71(0.42-1.2)$ & 0.2 & $0.60(0.29-1.27)$ & 0.184 & $0.75(0.40-1.41)$ & 0.377 \\
\hline Cardiovascular death & $136(1.9)$ & $56(1.6)$ & $17(2.2)$ & $0.71(0.41-1.23)$ & 0.219 & $0.60(0.28-1.29)$ & 0.190 & $0.76(0.40-1.45)$ & 0.406 \\
\hline MI & $610(8.5)$ & $307(8.5)$ & $45(5.8)$ & $1.48(1.08-2.02)$ & 0.014 & $1.05(0.73-1.50)$ & 0.800 & $0.97(0.68-1.38)$ & 0.856 \\
\hline Stroke & $29(0.4)$ & $13(0.4)$ & $1(0.1)$ & $2.81(0.37-21.5)$ & 0.298 & $2.40(0.27-21.18)$ & 0.43 & $4.50(0.56-35.99)$ & 0.156 \\
\hline TIA & $14(0.2)$ & $5(0.1)$ & $2(0.3)$ & $0.54(0.1-2.79)$ & 0.456 & $0.57(0.06-5.46)$ & 0.624 & $0.45(0.07-2.77)$ & 0.391 \\
\hline TVR & $87(1.2)$ & $52(1.4)$ & $10(1.3)$ & $1.12(0.57-2.21)$ & 0.733 & $1.16(0.57-2.39)$ & 0.678 & $1.20(0.58-2.49)$ & 0.619 \\
\hline ST definite & $57(0.8)$ & $36(1)$ & $6(0.8)$ & $1.30(0.55-3.08)$ & 0.553 & $1.55(0.63-3.85)$ & 0.341 & $1.68(0.67-4.21)$ & 0.265 \\
\hline Acute & $33(0.5)$ & $20(0.6)$ & $4(0.5)$ & $1.08(0.37-3.16)$ & 0.886 & $1.53(0.50-4.68)$ & 0.459 & $1.65(0.53-5.12)$ & 0.385 \\
\hline Subacute & $24(0.3)$ & $16(0.4)$ & $2(0.3)$ & $1.73(0.4-7.53)$ & 0.459 & $1.36(0.28-6.59)$ & 0.699 & $1.73(0.36-8.19)$ & 0.492 \\
\hline ST definite/probable & $80(1.1)$ & $45(1.2)$ & $9(1.2)$ & $1.08(0.53-2.21)$ & 0.83 & $1.28(0.57-2.86)$ & 0.554 & $1.33(0.62-2.87)$ & 0.467 \\
\hline Acute & $38(0.5)$ & $22(0.6)$ & $4(0.5)$ & $1.19(0.41-3.45)$ & 0.749 & $1.62(0.53-4.94)$ & 0.394 & $1.80(0.58-5.53)$ & 0.306 \\
\hline Subacute & $42(0.6)$ & $23(0.6)$ & $5(0.6)$ & $1.00(0.38-2.62)$ & 0.992 & $0.78(0.23-2.65)$ & 0.686 & $0.97(0.34-2.77)$ & 0.953 \\
\hline Bleeding & $873(12.1)$ & $391(10.8)$ & $137(17.5)$ & $0.62(0.51-0.75)$ & $<0.001$ & $0.64(0.5-0.83)$ & 0.001 & $0.64(0.50-0.80)$ & $<0.001$ \\
\hline BARC 1 & 427 (5.9) & $190(5.3)$ & $67(8.6)$ & $0.61(0.46-0.81)$ & 0.001 & $0.72(0.52-1.00)$ & 0.052 & $0.70(0.51-0.96)$ & 0.029 \\
\hline BARC 2 & $304(4.2)$ & $151(4.2)$ & $46(5.9)$ & $0.71(0.51-0.99)$ & 0.041 & $0.75(0.51-1.10)$ & 0.139 & $0.72(0.49-1.04)$ & 0.080 \\
\hline BARC 3 & $116(1.6)$ & $44(1.2)$ & $22(2.8)$ & $0.43(0.26-0.72)$ & 0.001 & $0.46(0.25-0.85)$ & 0.013 & $0.43(0.24-0.77)$ & 0.005 \\
\hline BARC 3a & $62(0.9)$ & $24(0.7)$ & $14(1.8)$ & $0.37(0.19-0.72)$ & 0.002 & $0.30(0.13-0.68)$ & 0.004 & $0.32(0.15-0.70)$ & 0.004 \\
\hline BARC 3b & $49(0.7)$ & $16(0.4)$ & $8(1.0)$ & $0.43(0.19-1.01)$ & 0.046 & $0.60(0.24-1.50)$ & 0.275 & $0.43(0.16-1.10)$ & 0.078 \\
\hline BARC $3 c$ & $5(0.1)$ & $4(0.1)$ & $0(0)$ & - & - & - & - & - & - \\
\hline BARC 4 & $5(0.1)$ & $1(0)$ & $0(0)$ & - & - & - & - & - & - \\
\hline BARC 5 & $21(0.3)$ & $5(0.1)$ & $2(0.3)$ & $0.54(0.10-2.79)$ & 0.456 & - & - & $0.57(0.09-3.63)$ & 0.553 \\
\hline BARC 5a & $15(0.2)$ & $4(0.1)$ & $2(0.3)$ & $0.43(0.08-2.36)$ & 0.319 & - & - & $0.38(0.05-2.73)$ & 0.336 \\
\hline BARC 5b & $6(0.1)$ & $1(0)$ & $0(0)$ & - & - & - & - & - & - \\
\hline BARC 3 or 5 & $137(1.9)$ & $49(1.4)$ & $24(3.1)$ & $0.44(0.27-0.72)$ & 0.001 & $0.47(0.26-0.85)$ & 0.013 & $0.44(0.25-0.77)$ & 0.004 \\
\hline BARC 3 or 5 access site & $51(0.7)$ & $19(0.5)$ & $9(1.2)$ & $0.46(0.21-1.01)$ & 0.047 & $0.57(0.23-1.37)$ & 0.209 & $0.44(0.18-1.07)$ & 0.070 \\
\hline BARC 3 or 5 nonaccess site & $86(1.2)$ & $30(0.8)$ & $15(1.9)$ & $0.43(0.23-0.8)$ & 0.006 & $0.42(0.19-0.95)$ & 0.036 & $0.45(0.22-0.91)$ & 0.027 \\
\hline BARC 2,3 or 5 & $441(6.1)$ & $200(5.5)$ & $70(9.0)$ & $0.62(0.47-0.81)$ & $<0.001$ & $0.64(0.46-0.89)$ & 0.009 & $0.61(0.45-0.84)$ & 0.002 \\
\hline BARC 2,3 or 5 access site & $237(3.3)$ & $105(2.9)$ & $34(4.4)$ & $0.67(0.45-0.98)$ & 0.04 & $0.66(0.42-1.03)$ & 0.067 & $0.60(0.39-0.93)$ & 0.021 \\
\hline BARC 2,3 or 5 nonaccess site & $204(2.8)$ & $95(2.6)$ & $36(4.6)$ & $0.57(0.39-0.84)$ & 0.004 & $0.65(0.40-1.03)$ & 0.069 & $0.65(0.42-1.00)$ & 0.049 \\
\hline TIMI major & $49(0.7)$ & $16(0.4)$ & $7(0.9)$ & $0.49(0.20-1.20)$ & 0.113 & $0.47(0.15-1.43)$ & 0.183 & $0.68(0.26-1.79)$ & 0.430 \\
\hline TIMI minor & $50(0.7)$ & $17(0.5)$ & $13(1.7)$ & $0.28(0.14-0.58)$ & $<0.001$ & $0.29(0.11-0.76)$ & 0.012 & $0.30(0.13-0.70)$ & 0.006 \\
\hline TIMI major/minor & $99(1.4)$ & $33(0.9)$ & $20(2.6)$ & $0.36(0.20-0.62)$ & $<0.001$ & $0.37(0.18-0.76)$ & 0.007 & $0.43(0.23-0.81)$ & 0.009 \\
\hline GUSTO severe & $42(0.6)$ & $16(0.4)$ & $6(0.8)$ & $0.58(0.23-1.47)$ & 0.245 & $0.67(0.20-2.20)$ & 0.505 & $0.75(0.27-2.11)$ & 0.590 \\
\hline GUSTO moderate & $42(0.6)$ & $16(0.4)$ & $9(1.2)$ & $0.38(0.17-0.87)$ & 0.017 & $0.29(0.11-0.80)$ & 0.017 & $0.29(0.12-0.74)$ & 0.010 \\
\hline GUSTO mild & $784(10.9)$ & $358(9.9)$ & $122(15.6)$ & $0.63(0.52-0.78)$ & $<0.001$ & $0.68(0.53-0.88)$ & 0.004 & $0.67(0.52-0.85)$ & 0.001 \\
\hline GUSTO moderate/severe & $84(1.2)$ & $32(0.9)$ & $15(1.9)$ & $0.46(0.25-0.85)$ & 0.011 & $0.42(0.20-0.88)$ & 0.022 & $0.46(0.23-0.92)$ & 0.027 \\
\hline $\begin{array}{l}\text { Composite of surgical access } \\
\text { site repair and blood transfusion }\end{array}$ & $103(1.4)$ & $36(1)$ & $16(2.0)$ & $0.49(0.27-0.88)$ & 0.014 & $0.42(0.20-0.88)$ & 0.022 & $0.39(0.20-0.76)$ & 0.006 \\
\hline Surgical access site repair & $17(0.2)$ & $5(0.1)$ & $4(0.5)$ & $0.27(0.07-1.01)$ & 0.036 & $0.25(0.06-1.15)$ & 0.075 & $0.18(0.04-0.79)$ & 0.023 \\
\hline Blood transfusion & $94(1.3)$ & $31(0.9)$ & $16(2.0)$ & $0.42(0.23-0.77)$ & 0.004 & $0.33(0.15-0.72)$ & 0.005 & $0.34(0.17-0.67)$ & 0.002 \\
\hline \multicolumn{10}{|l|}{ Distribution of BARC 3 or 5} \\
\hline Intracranial bleeding & $7(0.1)$ & $4(0.1)$ & $0(0)$ & - & - & - & - & - & - \\
\hline Pericardial bleeding & $28(0.4)$ & $11(0.3)$ & $3(0.4)$ & $0.79(0.22-2.84)$ & 0.722 & $0.81(0.16-4.23)$ & 0.807 & $1.02(0.26-4.05)$ & 0.976 \\
\hline Gastrointestinal bleeding & $27(0.4)$ & $6(0.2)$ & $5(0.6)$ & $0.26(0.08-0.85)$ & 0.016 & $0.18(0.03-0.93)$ & 0.041 & $0.16(0.04-0.64)$ & 0.009 \\
\hline Genito-urinary bleeding & $12(0.2)$ & $5(0.1)$ & $5(0.6)$ & $0.22(0.06-0.75)$ & 0.008 & $0.07(0.01-0.67)$ & 0.021 & $0.22(0.06-0.88)$ & 0.032 \\
\hline Access site bleeding & $49(0.7)$ & $19(0.5)$ & $8(1.0)$ & $0.51(0.22-1.17)$ & 0.108 & $0.65(0.26-1.64)$ & 0.36 & $0.51(0.20-1.28)$ & 0.150 \\
\hline Other bleeding & $10(0.1)$ & $3(0.1)$ & $2(0.3)$ & $0.32(0.05-1.94)$ & 0.194 & - & - & $0.11(0.01-1.51)$ & 0.098 \\
\hline
\end{tabular}




\begin{tabular}{|c|c|c|c|c|c|c|c|c|c|}
\hline & $\begin{array}{c}\text { All } \\
(\mathrm{N}=\mathbf{7 , 2 1 3})\end{array}$ & $\begin{array}{l}\text { UFH }+ \text { GPI } \\
(\mathbf{n}=\mathbf{7 8 1})\end{array}$ & $\begin{array}{l}\text { UFH Alone } \\
(n=2822)\end{array}$ & $\begin{array}{l}\text { Unadjusted } \\
\text { Rate Ratio } \\
(95 \% \mathrm{Cl})\end{array}$ & p Value & $\begin{array}{c}\text { Multivariable } \\
\text { Adjusted Rate Ratio } \\
(95 \% \mathrm{Cl})\end{array}$ & p Value & $\begin{array}{c}\text { Propensity } \\
\text { Score Adjusted } \\
\text { Rate Ratio } \\
(95 \% \mathrm{Cl})\end{array}$ & p Value \\
\hline Death, MI, stroke & $762(10.6)$ & $64(8.2)$ & $327(11.6)$ & $0.71(0.54-0.92)$ & 0.011 & $0.96(0.66-1.4)$ & 0.827 & $1.02(0.73-1.44)$ & 0.888 \\
\hline Death, MI, stroke, BARC 3 or 5 & 845 (11.7) & $82(10.5)$ & $362(12.8)$ & $0.82(0.64-1.04)$ & 0.101 & $1.09(0.77-1.52)$ & 0.633 & $1.15(0.84-1.57)$ & 0.377 \\
\hline $\begin{array}{l}\text { Death, MI, stroke, } \\
\text { BARC } 3 \text { or } 5 \text {, TVR, ST }\end{array}$ & 860 (11.9) & $83(10.6)$ & $367(13.0)$ & $0.82(0.64-1.04)$ & 0.096 & $1.05(0.75-1.47)$ & 0.785 & $1.10(0.81-1.50)$ & 0.526 \\
\hline Death & $142(2.0)$ & $18(2.3)$ & $65(2.3)$ & $1.00(0.59-1.69)$ & 0.998 & $0.84(0.22-3.18)$ & 0.799 & $0.82(0.41-1.63)$ & 0.565 \\
\hline Cardiovascular death & $136(1.9)$ & $17(2.2)$ & $63(2.2)$ & $0.98(0.57-1.67)$ & 0.926 & $0.59(0.12-2.89)$ & 0.515 & $0.74(0.36-1.51)$ & 0.401 \\
\hline MI & $610(8.5)$ & $45(5.8)$ & $258(9.1)$ & $0.63(0.46-0.86)$ & 0.004 & $0.99(0.66-1.47)$ & 0.959 & $1.11(0.76-1.64)$ & 0.581 \\
\hline Stroke & $29(0.4)$ & $1(0.1)$ & $15(0.5)$ & $0.24(0.03-1.82)$ & 0.134 & $0.11(0.01-1.50)$ & 0.098 & $0.15(0.02-1.37)$ & 0.093 \\
\hline TIA & $14(0.2)$ & $2(0.3)$ & $7(0.2)$ & $1.03(0.21-4.97)$ & 0.968 & $2.60(0.32-21.17)$ & 0.372 & $1.42(0.20-9.84)$ & 0.725 \\
\hline TVR & $87(1.2)$ & $10(1.3)$ & $25(0.9)$ & $1.45(0.69-3.01)$ & 0.322 & $1.08(0.47-2.45)$ & 0.859 & $0.86(0.35-2.14)$ & 0.747 \\
\hline ST definite & $57(0.8)$ & $6(0.8)$ & $15(0.5)$ & $1.45(0.56-3.73)$ & 0.443 & $1.09(0.38-3.17)$ & 0.871 & $0.78(0.24-2.52)$ & 0.683 \\
\hline Acute & $33(0.5)$ & $4(0.5)$ & $9(0.3)$ & $1.61(0.49-5.21)$ & 0.426 & $1.22(0.30-5.00)$ & 0.780 & $0.81(0.19-3.47)$ & 0.775 \\
\hline Subacute & $24(0.3)$ & $2(0.3)$ & $6(0.2)$ & $1.20(0.24-5.97)$ & 0.820 & $1.22(0.20-7.68)$ & 0.829 & $0.74(0.11-5.24)$ & 0.766 \\
\hline ST definite/probable & $80(1.1)$ & $9(1.2)$ & $26(0.9)$ & $1.25(0.59-2.67)$ & 0.562 & $1.13(0.43-2.93)$ & 0.805 & $1.02(0.39-2.65)$ & 0.969 \\
\hline Acute & $38(0.5)$ & $4(0.5)$ & $12(0.4)$ & $1.20(0.39-3.73)$ & 0.747 & $1.22(0.30-5.00)$ & 0.780 & $0.80(0.2-3.27)$ & 0.757 \\
\hline Subacute & $42(0.6)$ & $5(0.6)$ & $14(0.5)$ & $1.29(0.46-3.58)$ & 0.624 & $1.22(0.30-4.95)$ & 0.782 & $1.26(0.35-4.59)$ & 0.724 \\
\hline Bleeding & $873(12.1)$ & $137(17.5)$ & $345(12.2)$ & $1.43(1.18-1.75)$ & $<0.001$ & $1.27(0.97-1.68)$ & 0.084 & $1.27(0.97-1.66)$ & 0.082 \\
\hline BARC 1 & $427(5.9)$ & $67(8.6)$ & $170(6.0)$ & $1.42(1.07-1.89)$ & 0.014 & $1.14(0.79-1.65)$ & 0.471 & $1.08(0.75-1.55)$ & 0.687 \\
\hline BARC 2 & $304(4.2)$ & $46(5.9)$ & $107(3.8)$ & $1.55(1.10-2.19)$ & 0.012 & $1.45(0.95-2.20)$ & 0.084 & $1.48(0.95-2.30)$ & 0.084 \\
\hline BARC 3 & $116(1.6)$ & $22(2.8)$ & $50(1.8)$ & $1.59(0.96-2.63)$ & 0.067 & $1.66(0.86-3.18)$ & 0.129 & $1.84(0.98-3.46)$ & 0.059 \\
\hline BARC 3a & $62(0.9)$ & $14(1.8)$ & $24(0.9)$ & $2.11(1.09-4.07)$ & 0.023 & $3.07(1.20-7.86)$ & 0.019 & $2.82(1.24-6.44)$ & 0.014 \\
\hline BARC 3b & $49(0.7)$ & $8(1.0)$ & $25(0.9)$ & $1.16(0.52-2.56)$ & 0.721 & $0.94(0.35-2.47)$ & 0.892 & $1.10(0.41-2.93)$ & 0.851 \\
\hline BARC $3 c$ & $5(0.1)$ & $0(0)$ & $1(0)$ & - & - & - & - & - & - \\
\hline BARC 4 & $5(0.1)$ & $0(0)$ & $4(0.1)$ & - & - & - & - & - & - \\
\hline BARC 5 & $21(0.3)$ & $2(0.3)$ & $14(0.5)$ & $0.52(0.12-2.27)$ & 0.373 & - & - & $0.25(0.05-1.34)$ & 0.105 \\
\hline BARC 5a & $15(0.2)$ & $2(0.3)$ & $9(0.3)$ & $0.80(0.17-3.72)$ & 0.778 & - & - & $0.37(0.06-2.28)$ & 0.284 \\
\hline BARC 5b & $6(0.1)$ & $0(0)$ & $5(0.2)$ & - & - & - & - & - & - \\
\hline BARC 3 or 5 & $137(1.9)$ & $24(3.1)$ & $64(2.3)$ & $1.35(0.85-2.17)$ & 0.203 & $1.41(0.74-2.68)$ & 0.293 & $1.33(0.74-2.41)$ & 0.342 \\
\hline BARC 3 or 5 access site & $51(0.7)$ & $9(1.2)$ & $23(0.8)$ & $1.41(0.65-3.06)$ & 0.376 & $1.49(0.57-3.91)$ & 0.417 & $1.86(0.71-4.84)$ & 0.205 \\
\hline BARC 3 or 5 nonaccess site & $86(1.2)$ & $15(1.9)$ & $41(1.5)$ & $1.32(0.73-2.39)$ & 0.353 & $1.35(0.58-3.13)$ & 0.482 & $1.10(0.52-2.32)$ & 0.805 \\
\hline BARC 2,3 or 5 & $441(6.1)$ & $70(9.0)$ & $171(6.1)$ & $1.48(1.12-1.95)$ & 0.005 & $1.43(1.00-2.05)$ & 0.051 & $1.44(1.01-2.07)$ & 0.047 \\
\hline BARC 2,3 or 5 access site & $237(3.3)$ & $34(4.4)$ & $98(3.5)$ & $1.25(0.85-1.85)$ & 0.255 & $1.31(0.82-2.12)$ & 0.262 & $1.53(0.94-2.49)$ & 0.089 \\
\hline BARC 2,3 or 5 nonaccess site & $204(2.8)$ & $36(4.6)$ & $73(2.6)$ & $1.78(1.20-2.66)$ & 0.004 & $1.65(0.98-2.79)$ & 0.062 & $1.32(0.79-2.21)$ & 0.285 \\
\hline TIMI major & $49(0.7)$ & $7(0.9)$ & $26(0.9)$ & $0.97(0.42-2.24)$ & 0.948 & $0.66(0.23-1.93)$ & 0.447 & $0.66(0.24-1.83)$ & 0.424 \\
\hline TIMI minor & $50(0.7)$ & $13(1.7)$ & $20(0.7)$ & $2.35(1.17-4.72)$ & 0.014 & $2.61(0.92-7.43)$ & 0.072 & $2.66(1.09-6.51)$ & 0.032 \\
\hline TIMI major/minor & $99(1.4)$ & $20(2.6)$ & $46(1.6)$ & $1.57(0.93-2.66)$ & 0.089 & $1.37(0.66-2.84)$ & 0.404 & $1.38(0.70-2.70)$ & 0.351 \\
\hline GUSTO severe & $42(0.6)$ & $6(0.8)$ & $20(0.7)$ & $1.08(0.44-2.7)$ & 0.862 & $0.86(0.25-2.99)$ & 0.816 & $0.83(0.27-2.53)$ & 0.746 \\
\hline GUSTO moderate & $42(0.6)$ & $9(1.2)$ & $17(0.6)$ & $1.91(0.85-4.29)$ & 0.109 & $3.11(1.03-9.43)$ & 0.044 & $3.04(1.13-8.21)$ & 0.028 \\
\hline GUSTO mild & $784(10.9)$ & $122(15.6)$ & $304(10.8)$ & $1.45(1.18-1.79)$ & $<0.001$ & $1.22(0.92-1.62)$ & 0.167 & $1.23(0.92-1.62)$ & 0.158 \\
\hline GUSTO moderate/severe & $84(1.2)$ & $15(1.9)$ & $37(1.3)$ & $1.46(0.80-2.67)$ & 0.21 & $1.73(0.76-3.94)$ & 0.192 & $1.63(0.77-3.43)$ & 0.199 \\
\hline $\begin{array}{l}\text { Composite of surgical access site } \\
\text { repair and blood transfusion }\end{array}$ & $103(1.4)$ & $16(2.0)$ & $51(1.8)$ & $1.13(0.65-1.99)$ & 0.661 & $1.29(0.61-2.72)$ & 0.51 & $1.52(0.76-3.05)$ & 0.233 \\
\hline Surgical access site repair & $17(0.2)$ & $4(0.5)$ & $8(0.3)$ & $1.81(0.54-6.00)$ & 0.327 & $2.54(0.49-13.23)$ & 0.267 & $2.91(0.65-12.95)$ & 0.161 \\
\hline Blood transfusion & $94(1.3)$ & $16(2.0)$ & $47(1.7)$ & $1.23(0.7-2.17)$ & 0.474 & $1.39(0.65-2.97)$ & 0.400 & $1.65(0.82-3.33)$ & 0.163 \\
\hline \multicolumn{10}{|l|}{ Distribution of BARC 3 or 5} \\
\hline Intracranial bleeding & $7(0.1)$ & $0(0)$ & $3(0.1)$ & - & - & - & - & - & - \\
\hline Pericardial bleeding & $28(0.4)$ & $3(0.4)$ & $14(0.5)$ & $0.77(0.22-2.69)$ & 0.687 & $0.92(0.15-5.78)$ & 0.928 & $0.52(0.12-2.27)$ & 0.384 \\
\hline Gastrointestinal bleeding & $27(0.4)$ & $5(0.6)$ & $16(0.6)$ & $1.13(0.41-3.08)$ & 0.812 & $1.30(0.34-5.05)$ & 0.703 & $0.99(0.29-3.36)$ & 0.985 \\
\hline Genito-urinary bleeding & $12(0.2)$ & $5(0.6)$ & $2(0.1)$ & $9.03(1.75-46.56)$ & 0.001 & - & - & $11.14(1.69-73.65)$ & 0.012 \\
\hline Access site bleeding & $49(0.7)$ & $8(1.0)$ & $22(0.8)$ & $1.31(0.58-2.95)$ & 0.507 & $1.41(0.51-3.90)$ & 0.505 & $1.62(0.59-4.44)$ & 0.345 \\
\hline Other bleeding & $10(0.1)$ & $2(0.3)$ & $5(0.2)$ & $1.45(0.28-7.45)$ & 0.658 & $0.87(0.03-27.51)$ & 0.936 & $1.88(0.17-21.24)$ & 0.610 \\
\hline
\end{tabular}


FIGURE 1 Coprimary Composite Study Outcomes at 30 Days

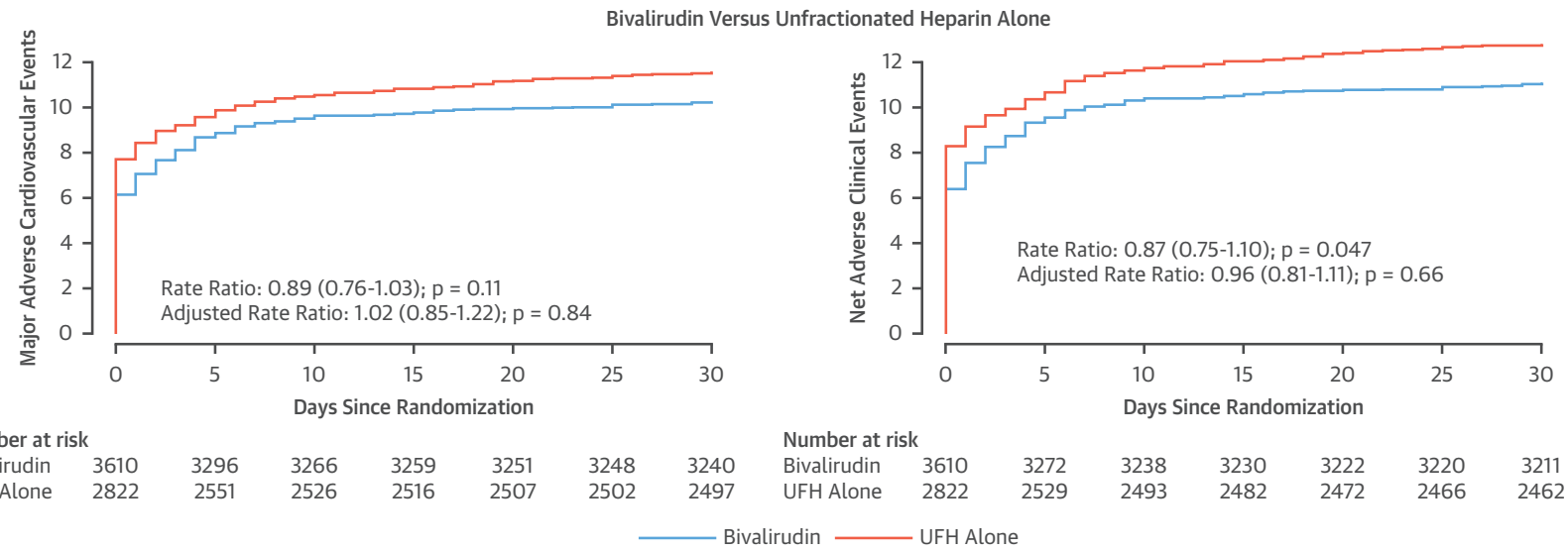

\begin{tabular}{|c|c|c|c|c|c|c|c|c|c|c|c|c|c|c|c|}
\hline Number at & & & & & & & & Number at & & & & & & & \\
\hline Bivalirudin & 3610 & 3296 & 3266 & 3259 & 3251 & 3248 & 3240 & Bivalirudin & 3610 & 3272 & 3238 & 3230 & 3222 & 3220 & 3211 \\
\hline UFH Alone & 2822 & 2551 & 2526 & 2516 & 2507 & 2502 & 2497 & UFH Alone & 2822 & 2529 & 2493 & 2482 & 2472 & 2466 & 2462 \\
\hline
\end{tabular}
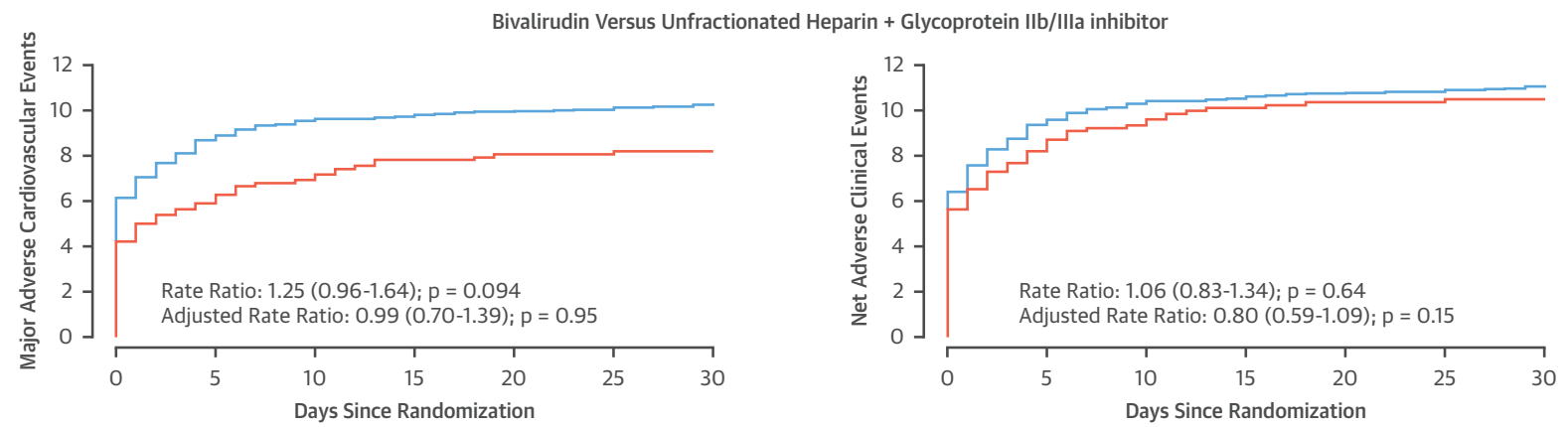

Number at risk Number at risk

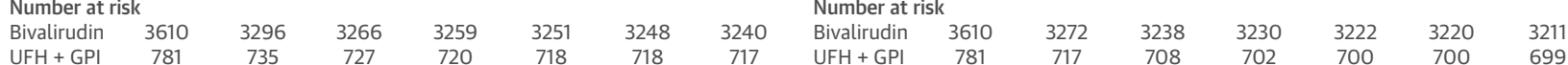

Bivalirudin $-\mathrm{UFH}+\mathrm{GPI}$

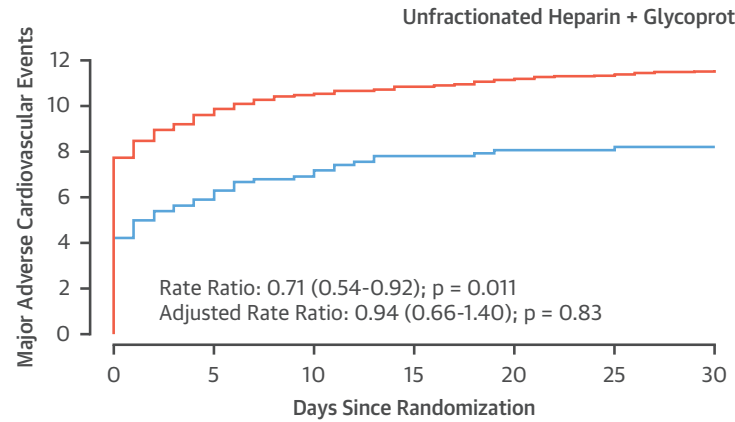

\section{inhibitor Versus Unfractionated Heparin Alone}

Number at risk

$\begin{array}{llllllll}\text { UFH + GPI } & 781 & 735 & 727 & 720 & 718 & 718 & 717\end{array}$

$\begin{array}{llllllll}\text { UFH Alone } & 2822 & 2551 & 2526 & 2516 & 2507 & 2502 & 2497\end{array}$

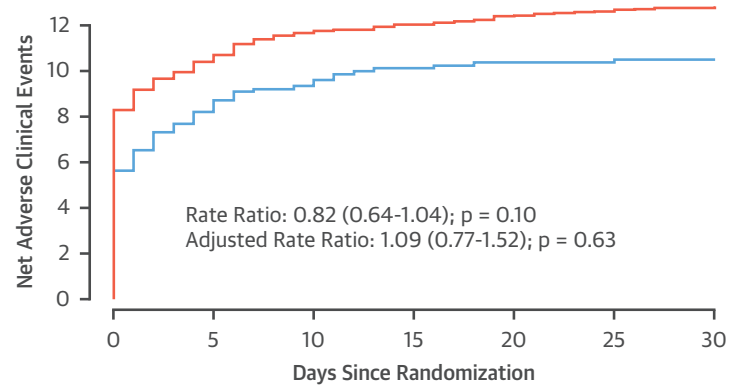

Number at risk

$\begin{array}{llllllll}\text { UFH + GPI } & 781 & 717 & 708 & 702 & 700 & 700 & 699\end{array}$

$\begin{array}{lllllll}\text { UFH Alone } & 2822 & 2529 & 2493 & 2482 & 2472 & 2466\end{array}$

$-\mathrm{UFH}+\mathrm{GPI}$

UFH Alone

(Left) The cumulative incidence of the coprimary outcome of major adverse cardiovascular events and (right) net adverse clinical events up to 30 days, (top) among patients who received bivalirudin versus unfractionated heparin (UFH) alone, (middle) bivalirudin versus UFH plus glycoprotein IIb/IIla inhibitors (GPI), and (bottom) UFH plus planned GPI versus UFH alone.

genito-urinary hemorrhages compared with UFH+GPI (Tables 1 and 2).

Transfusion rates and need for surgical access site repair were also reduced in the bivalirudin group
(Tables 1 and 2). Conversely, bleeding complications, mainly genito-urinary hemorrhages that fulfilled Bleeding Academic Research Consortium grade 3A, TIMI minor, or GUSTO moderate criteria were 
CENTRAL ILLUSTRATION Bivalirudin or Heparin in ACS: Forest Plots of Main Outcomes

\section{A Bivalirudin versus UFH alone}

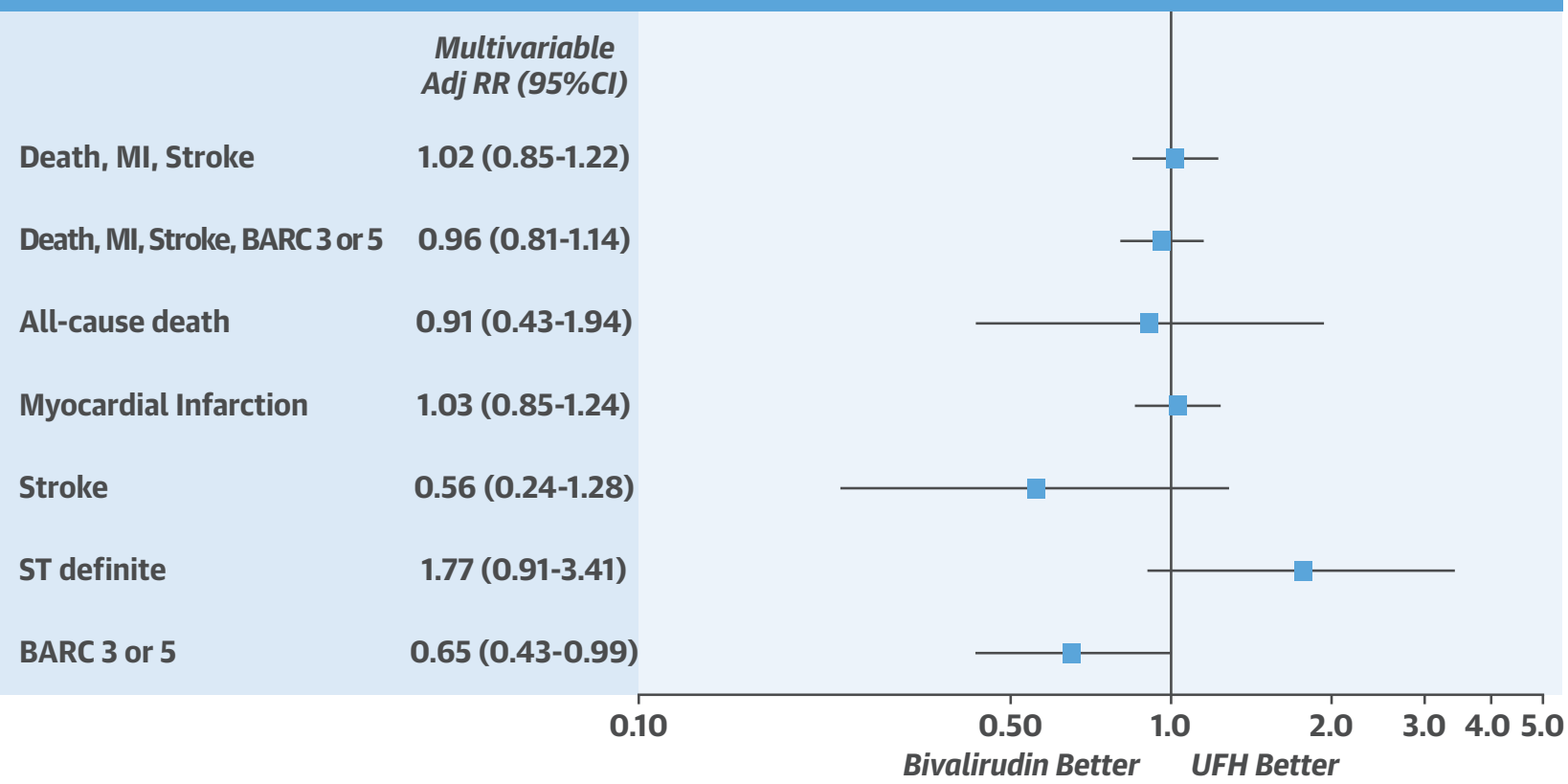

\section{B Bivalirudin versus UFH + GPI}

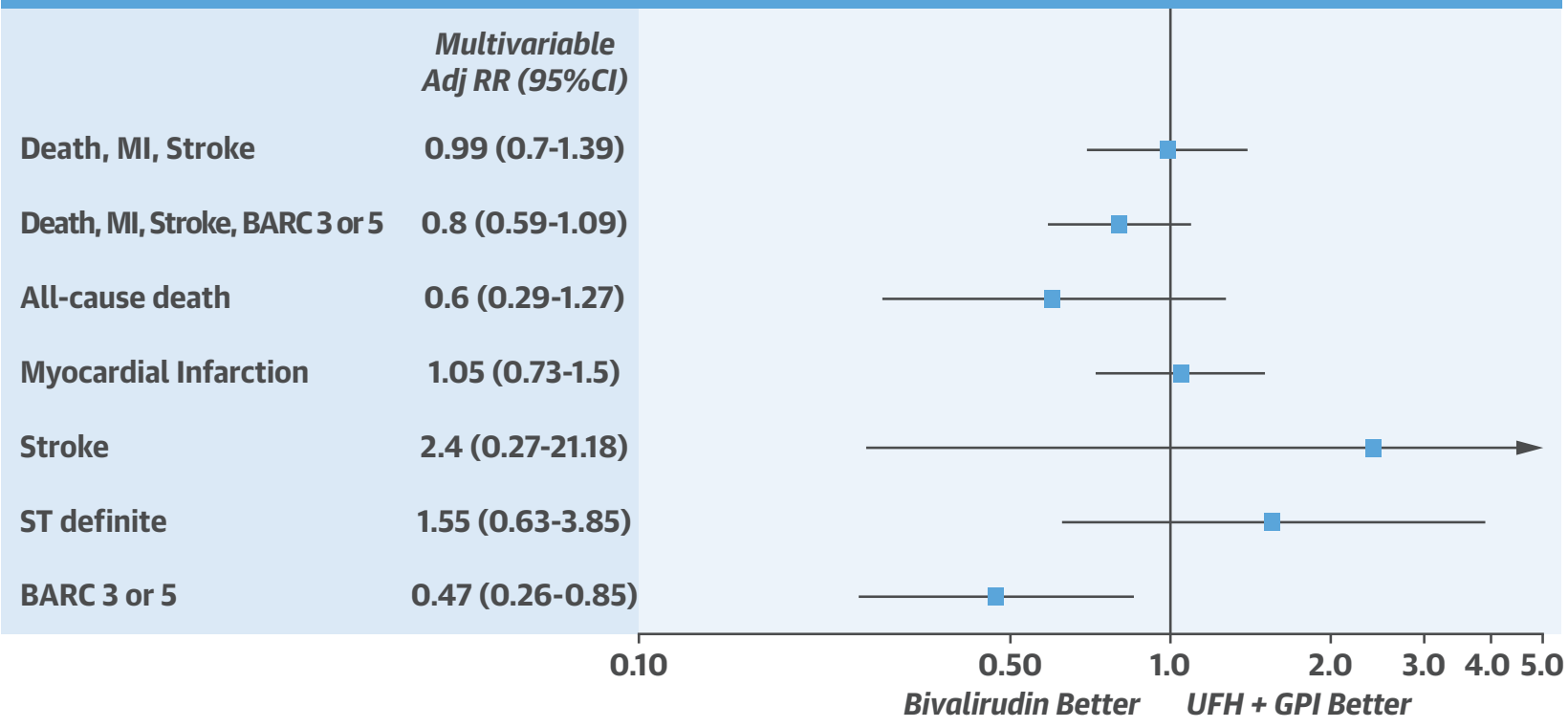

Gargiulo, G. et al. J Am Coll Cardiol. 2018;71(11):1231-42.

Multivariable adjusted rate ratios (RR) of main outcomes at 30 days for (A) bivalirudin versus unfractionated heparin (UFH) alone or (B) bivalirudin versus UFH plus planned glycoprotein IIb/IIla inhibitors (GPIs) comparisons. BARC = Bleeding Academic Research Consortium; $\mathrm{Cl}=$ confidence interval; $\mathrm{MI}=$ myocardial infarction; ST $=$ stent thrombosis. 
increased in the UFH+GPI group compared with UFH alone (Table 3).

STRATIFIED AND PROPENSITY-MATCHING ANALYSES. We performed stratified analyses of the main clinical outcomes in the 3 comparisons, and we observed that, after adjustment, results remained consistent across UFH dose subgroups (Online Table 4). We also stratified major bleeding events by access site and observed that bivalirudin consistently reduced access site and nonaccess site bleeding compared with UFH groups, irrespective of the randomly allocated arterial access (Online Table 5).

After propensity-score matching was applied to the MATRIX population, 2,698 matched pairs of patients were identified for the comparison of bivalirudin versus UFH alone; there were 747 pairs of patients for the comparison bivalirudin versus UFH+GPI group and 578 pairs of patients for the comparison of UFH alone versus UFH+GPI. This model, developed to account for the nonrandomized use of GPI in the UFH arm, showed good discrimination and calibration (area under the curve: 0.85; 95\% confidence interval: 0.83 to 0.87 ; HosmerLemeshow: $\mathrm{p}=0.352$ ) (Online Figure 1). Post-match standardized differences for almost all measured covariates were $<10 \%$, which suggested substantial balance across the groups (Online Figures 2 to 4, Online Tables 6 to 11). Results of clinical outcomes at 30 days remained consistent with primary adjusted analyses (Online Tables 12 to 14), which confirmed a beneficial effect of bivalirudin versus UFH alone with respect to fatal and major bleeding across all adopted bleeding classifications.

\section{DISCUSSION}

The salient findings of this pre-specified analysis of the MATRIX trial can be summarized as follows:

1. The rates of MACEs and NACEs were not significantly lower among those who received bivalirudin compared with among those who received unfractionated heparin alone or with planned GPI at the time of PCI.

2. Compared with UFH and UFH+GPI groups, bivalirudin consistently reduced major bleeding, including fatal and nonaccess site-related events, as well as transfusion rates and need for surgical access site repair. This observation was consistent with the multivariable, propensity score-adjusted and propensity score-matched analyses. Although ST trended higher and mortality lower with bivalirudin compared with UFH alone or UFH+GPI, none of the single components of the primary composite endpoints, apart from bleeding, differed at a statistically significant level.

After initial studies, bivalirudin was approved and used during PCI due to the reduction of bleeding complications and similar ischemic risks compared with UFH+GPI. Although an excess of acute ST has been consistently noted in STEMI patients treated with bivalirudin compared with UFH+GPI (11), an early mortality benefit in the bivalirudin arm of the pivotal HORIZONS-AMI (Harmonizing Outcomes with Revascularization and Stents in Acute Myocardial Infarction) trial has reinforced the notion in the community that bleeding prevention has the potential to affect mortality endpoints at least as much as ischemic events (12). However, the routine use of GPI on top of UFH has been regarded as unwarranted, and it may have further increased the bleeding risk in the comparator arms of approval bivalirudin studies.

In the last decade, the introduction of potent oral $\mathrm{P}_{2} \mathrm{Y}_{12}$ inhibitors and the diffusion of the radial access have reduced the rates of PCI-related ischemic and bleeding events, respectively, thus, further questioning the need for routine or even selective use of GPI in most ACS cases. Most recent trials, including EUROMAX (6) and BRIGHT (7), have shown benefits in terms of major bleeding reduction related to bivalirudin use, irrespective of GPI use in the UFH arm. In opposite, the HEAT-PPCI trial showed that heparin compared with bivalirudin reduced the incidence of MACEs and ST, with no increase in bleeding complications (8). The most recent VALIDATESWEDEHEART study aimed at addressing these uncertainties by comparing bivalirudin versus UFH alone in ACS patients (matched STEMI and NSTEMI) who underwent PCI by radial access site and treatment with new $\mathrm{P}_{2} \mathrm{Y}_{12}$ inhibitors (9). The trial enrolled 6,006 patients and showed a null effect of bivalirudin versus UFH with respect to the composite primary endpoint, including ischemic and bleeding events, as well as for each individual endpoint at 6 months. The absence of clear bleeding benefits with bivalirudin was attributed to the high rate of radial access ( $\approx 90 \%)$ and negligible use of GPI, which were restricted to bailout situations $(\approx 3 \%)$ (9). However, there were additional factors beyond radial access and no planned use of GPI that might have contributed to explaining the lack of bleeding benefit in this study. A trend in favor of bivalirudin for bleeding endpoints was noted at 30 days, a time frame that seems more suitable to capturing the true value of a purely periprocedural antithrombotic compound. In addition, the allowance of UFH administration both before (up to 5,000 $\mathrm{U}$ ) and in the catheterization 
laboratory (up to 3,000 $\mathrm{U}$ ) in the bivalirudin arm might have contributed to bias the results towards the null. In the MATRIX trial, planned GPI was allowed in the UFH arm and actually used in less than one-quarter of patients. Moreover, by study design, an equal and random proportion of patients were intervened upon by either radial or femoral access.

We found a consistent effect of bivalirudin in mitigating the bleeding risk across groups, largely from nonaccess site-related complications. This observation suggested that differences in study design and/or study populations might explain the apparently inconsistent effect of bivalirudin on bleeding endpoints beyond the selected access site or planned GPI use.

However, similar to VALIDATE-SWEDEHEART and other previous studies, no clear effect of bivalirudin on the primary composite endpoints was noted, irrespective of concomitant use of GPI in the UFH arm. Moreover, no clear effect of bivalirudin on ST or mortality rates was identified compared with UFH alone. This might reflect the limited study power to assess a treatment effect for relatively rare endpoints, which was further amplified by the need to apply multivariable analytical tools to account for the nonrandomized nature of GPI or the lack of a true treatment effect.

The dose of UFH in the control arms of available studies is also worth discussing. In the HEAT-PPCI study, UFH was dosed at $70 \mathrm{U} / \mathrm{kg}$, which might have contributed to the absence of bleeding advantages with bivalirudin (8). In contrast, the use of $100 \mathrm{U} / \mathrm{kg}$ UFH might have inflated the risks of bleeding in the UFH arms of the EUROMAX and BRIGHT studies (5-7). However, in the MATRIX trial, UFH was administered at a mean dose of $78 \mathrm{U} / \mathrm{kg}$ in the control group, and our results remained entirely consistent at stratified analyses by low and high UFH doses. Thus, our present results did not support the interpretation that differences across studies might be reconciled by simply taking the different recommended UFH doses into account.

The present study added to previous evidence and supported the concept that bivalirudin does not provide benefits in terms of composite endpoints, including ischemic or ischemic and bleeding events. However, our results suggested benefits for bivalirudin in terms of bleeding risk mitigation, in either femoral or radial access. Thus, in addition to the well-established recommendation for patients with heparin-induced thrombocytopenia, bivalirudin should be considered as an alternative to UFH, particularly in high risk of bleeding patients.
Ultimately, an individual patient data meta-analysis of all major bivalirudin studies could shed new light on the merits and limits of bivalirudin versus UFH with or without GPI in current practice (3).

STUDY LIMITATIONS. Although this was a prespecified analysis, use of planned GPI was left to the discretion of the physician, thus, generating 3 groups that were imbalanced in number and characteristics. The use of multivariable adjustment and propensity score matching successfully eliminated measured confounders. However, residual unmeasured confounding could not be excluded. The value of postPCI bivalirudin infusion was not analyzed due to the need to account for 2 different nonrandomly allocated post-PCI bivalirudin regimens, which were seemingly associated with different study results at univariate analysis (1) and required further dedicated multivariable investigations.

\section{CONCLUSIONS}

Among patients with ACS who underwent invasive treatment, the planned use of GPIs in the control group did not affect the comparative effectiveness and safety profile of bivalirudin versus UFH. Consistent with the main study results, bivalirudin did not decrease the rates of the coprimary endpoints compared with UFH alone, but it did remain associated with consistent bleeding benefits, largely coming from major episodes, which were unrelated to the access site. The effect of bivalirudin versus UFH alone on more infrequent endpoints, such as ST or fatal events requires further investigation.

ADDRESS FOR CORRESPONDENCE: Dr. MarCO Valgimigli, Department of Cardiology, Bern University Hospital, Freiburgstrasse 4, CH-3010, Bern, Switzerland. E-mail: marco.valgimigli@insel.ch.

\section{PERSPECTIVES}

\section{COMPETENCY IN PATIENT CARE AND PROCEDURAL}

SKILLS: In patients with ACSs who underwent percutaneous revascularization, bivalirudin was associated with comparable efficacy and less bleeding compared with UFH, regardless of access site or concurrent therapy with GPIs.

TRANSLATIONAL OUTLOOK: Additional investigation is needed to compare bivalirudin against UFH on other endpoints, such as stent thrombosis and mortality, and to assess the cost-effectiveness of these strategies. 


\section{REFEREN CES}

1. Valgimigli $M$, Frigoli $E$, Leonardi $S$, et al. BivaLirudin or unfractionated heparin in acute coronary syndromes. N Engl J Med 2015;373:997-1009.

2. Gargiulo G, Moschovitis $A$, Windecker $S$, Valgimigli M. Developing drugs for use before, during and soon after percutaneous coronary intervention. Expert Opin Pharmacother 2016;17: 803-18.

3. Stone GW. Procedural anticoagulation in myocardial infarction. N Engl J Med 2017;377: 1198-200.

4. Valgimigli $M$, Gargiulo G. Bivalirudin in current practice: melius abundare quam deficere? J Am Coll Cardiol Intv 2016;9:1321-3.

5. Steg PG, van 't Hof $A$, Hamm CW, et al. Bivalirudin started during emergency transport for primary PCI. N Engl J Med 2013;369:2207-17.

6. Zeymer U, van 't Hof A, Adgey J, et al. Bivalirudin is superior to heparins alone with bailout GP Ilb/Illa inhibitors in patients with ST-segment elevation myocardial infarction transported emergently for primary percutaneous coronary intervention: a pre-specified analysis from the EUROMAX trial. Eur Heart J 2014;35:2460-7.

7. Han Y, Guo J, Zheng Y, et al. Bivalirudin vs heparin with or without tirofiban during primary percutaneous coronary intervention in acute myocardial infarction: the BRIGHT randomized clinical trial. JAMA 2015;313 1336-46.

8. Shahzad A, Kemp I, Mars C, et al. Unfractio nated heparin versus bivalirudin in primary percutaneous coronary intervention (HEAT-PPCI): an open-label, single centre, randomised controlled trial. Lancet 2014;384:1849-58.

9. Erlinge $D$, Omerovic $E$, Frobert $O$, et al. Bivalirudin versus heparin monotherapy in myocardial infarction. N Engl J Med 2017;377: 1132-42.

10. Valgimigli $M$, Gagnor $A$, Calabro $P$, et al Radial versus femoral access in patients with acute coronary syndromes undergoing invasive management: a randomised multicentre trial. Lancet 2015;385:2465-76.

11. Capodanno D, Gargiulo G, Capranzano P, Mehran R, Tamburino C, Stone GW. Bivalirudin versus heparin with or without glycoprotein IIb/IIla inhibitors in patients with STEMI undergoing primary PCl: an updated meta-analysis of 10,350 patients from five randomized clinical trials. Eur Heart J Acute Cardiovasc Care 2016;5:253-62.

12. Stone GW, Witzenbichler $B$, Guagliumi $G$, et al. Bivalirudin during primary $\mathrm{PCl}$ in acute myocardial infarction. N Engl J Med 2008;358:2218-30.

KEY WORDS acute coronary syndrome, bivalirudin, GP IIb/IIla inhibitor, heparin, MATRIX

APPENDIX For supplemental tables and figures, please see the online version of this paper. 